\title{
Emerging role of the Hippo pathway in autophagy
}

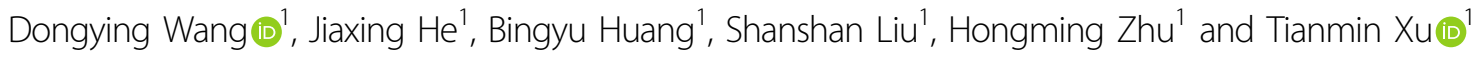

\begin{abstract}
Autophagy is a dynamic circulatory system that occurs in all eukaryotic cells. Cytoplasmic material is transported to lysosomes for degradation and recovery through autophagy. This provides energy and macromolecular precursors for cell renewal and homeostasis. The Hippo-YAP pathway has significant biological properties in controlling organ size, tissue homeostasis, and regeneration. Recently, the Hippo-YAP axis has been extensively referred to as the pathophysiological processes regulating autophagy. Understanding the cellular and molecular basis of these processes is crucial for identifying disease pathogenesis and novel therapeutic targets. Here we review recent findings from Drosophila models to organisms. We particularly emphasize the regulation between Hippo core components and autophagy, which is involved in normal cellular regulation and the pathogenesis of human diseases, and its application to disease treatment.
\end{abstract}

\section{Facts}

- The Hippo pathway and autophagy have complex and reciprocal interactions. These bidirectional links coordinate the autophagic flux to the overall microenvironmental signal and regulate homeostasis and tumorigenesis.

- Autophagy is involved in the biological effects of the Hippo pathway and vice versa. Hippo-mediated modalities profoundly influence autophagic flux and are extensively involved in the intracellular quality control, tissue homeostasis, regeneration, development, and differentiation.

- The association between the Hippo pathway and autophagy is relevant to the pathogenesis of a wide range of human diseases, from metabolic and neurodegenerative diseases, cardiovascular diseases to a variety of human solid tumors.

- The emerging link between the Hippo pathway and autophagy means that targeting the Hippo-YAPautophagy axis may provide new insights to prevent or promote autophagy in a variety of contexts, influencing metabolic reprogramming, cellular

Correspondence: Tianmin Xu (xutianmin@126.com)

'Department of Obstetrics and Gynecology, The Second Hospital, Jilin University, 218 Zi Qiang Street, Changchun, Jilin 130000, China Edited by G. Blandino mechanical signals, mitochondrial quality control, and YAP/TAZ transcriptional activity.

\section{Open questions}

- How are the Hippo pathway and autophagy interconnected, and what are the implications of these bidirectional links in homeostasis and tumorigenesis?

- How prevalent is the Hippo-autophagy axis and what is its significance in disease development?

- Will the emerging link between the Hippo pathway and autophagy make the role of targeted autophagy in cancer therapy clearer?

\section{Introduction}

Autophagy (also known as macroautophagy) is an accommodative process that occurs in different forms of cell stresses, including starvation, hypoxia, infection, high reactive oxygen content, and endoplasmic reticulum stress $^{1}$. During this process, cells capture intracellular proteins and organelles, and transport them to lysosomes for degradation and export the products of autophagic degradation from lysosomes to the cytoplasm for recycling $^{2,3}$. The first genetic screening of autophagy was conducted in Ohsumi's laboratory, which analyzed this process in yeast and identified 15 autophagy-related 


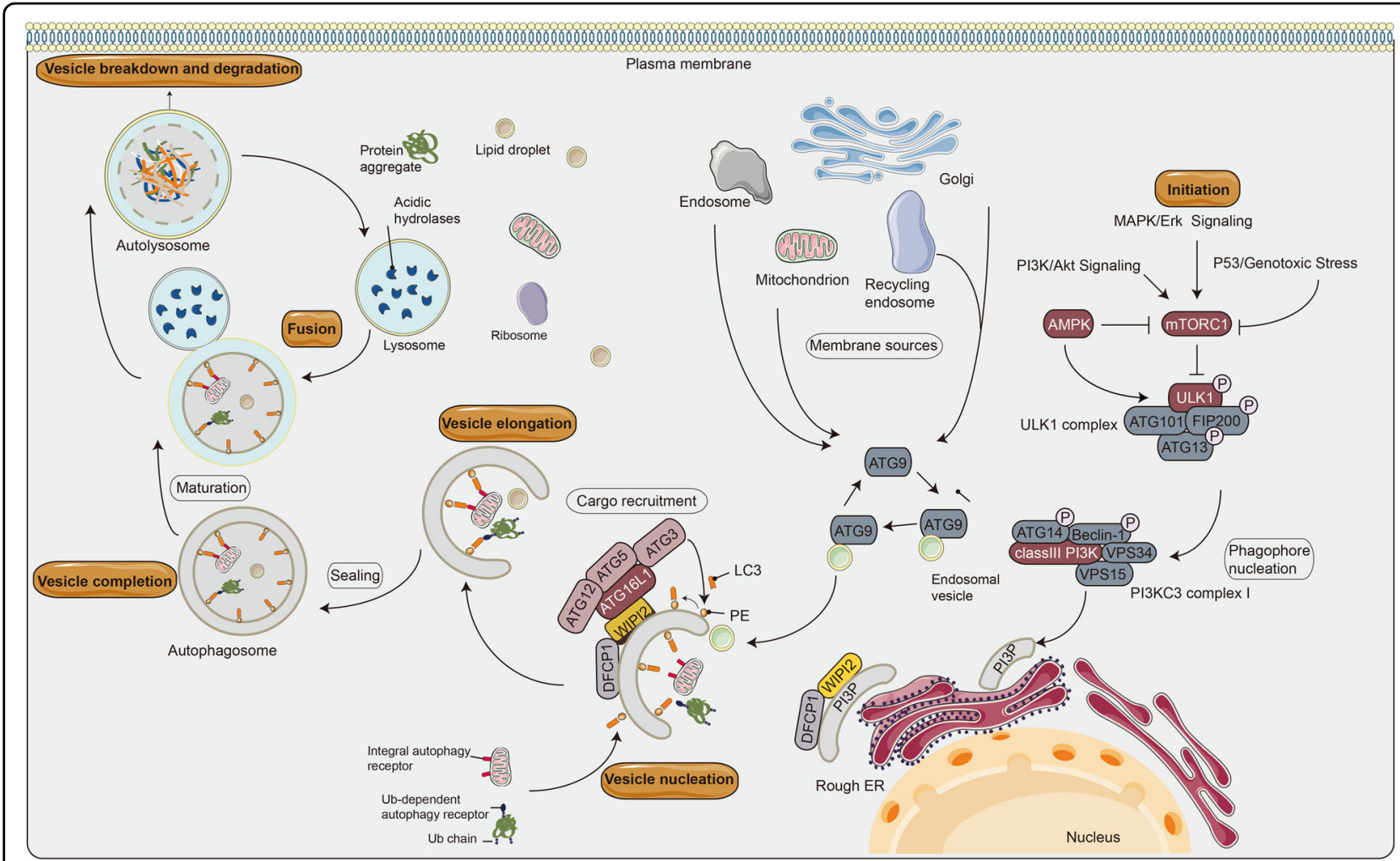

Fig. 1 Schematic diagram of the autophagy process in mammalian cells. The mTOR complex 1 (mTORC1) contributes to the initiation of autophagy, integrates upstream signals such as PI3K/Akt pathway, AMPK, P53, and Bcl-2 protein family, which play different regulatory roles in autophagy ${ }^{135}$. The ULK1 complex induces vesicle nucleation and translocates to a characteristic endoplasmic reticulum (ER) structure called omegasome, where it phosphorylates PI3KC3 complex I to produce phosphatidylinositol-3-phosphate (PI3P) in omegasome. Specifically, Beclin1, a BCl-2-homology (BH)-3 domain-only protein, is phosphorylated by ULK1 and acts as a scaffold for the PI3KC3 complex I, which facilitates localization of autophagy proteins to the phagophore. Atg9 is a transmembrane protein, which participates in the early stage of phagophore formation. Pl3P recruits specific autophagy effectors, such as WIPIs (mammalian homolog of yeast Atg18) and zinc finger FYVE-type containing 1 (DFCP1). WIPIs directly binds to ATG16L1 under the regulation of the ubiquitin-like conjugation system to form the ATG12-ATG5-ATG16L1 complex and LC3 (mammalian homolog of yeast Atg8)-phosphatidylethanolamine (PE) binding. Ultimately, the isolation membrane is elongated and closed to form the autophagosome ${ }^{136}$. This binding reaction results in the conversion of LC3-I to LC3-II, a common autophagosome marker. When the autophagosome matures, it sheds the ATG proteins and fuses with the lysosome to produce autophagolysosome. Both the inner membrane of the autophagic vesicle and the luminal contents are degraded by lysosomal hydrolases (cathepsins B, D, and L). The resulting monomer molecules (such as amino acids and lipids) are recycled into the cytoplasm for reuse ${ }^{137}$. The pointed and blunt arrowheads indicate activation and inhibitory interactions, respectively. Ub, ubiquitin.

proteins (ATGs $)^{4}$. So far, over 30 ATGs have been identified $^{5}$. The autophagy pathway is frequently divided into various individual stages: initiation, vesicle nucleation, elongation of the autophagy membrane, fusion with lysosomes, and degradation of intravesicular products (Fig. 1) ${ }^{6}$. Previously, autophagy has been considered a non-selective process. Recent studies have shown that autophagy can selectively eliminate harmful cytosols such as invading pathogens, dysfunctional organelles, and protein aggregates (called selective autophagy, including lipophagy, mitophagy, xenophagy, and aggrephagy), thereby contributing to the protection of cells in various environmental and metabolic stress ${ }^{3,7}$. Autophagy is strongly associated with neurodegeneration, cancer, metabolic diseases, immune and heart diseases, especially the role of autophagy in cancer ${ }^{7}$. Autophagy plays a dual role in cancer. Under tumorigenesis pressure, autophagy can clear oncogenic protein substrates and toxic unfolded proteins, inhibiting tissue damage and genomic instability $^{8-10}$. Conversely, after tumor formation, increased autophagic flux often allows tumor cells to survive and grow $^{9,11}$. This makes autophagy an interesting target for pharmacologists and clinicians.

The Hippo-Yes-associated protein (YAP) pathway is an evolutionarily conserved pathway that controls organ size and tissue homeostasis ${ }^{12}$. The core kinase cassettes of the mammalian Hippo-YAP pathway consist of the mammalian sterile 20-like protein kinase 1 (STK3/MST2 and STK4/MST1) and an adapter protein, salvador family WW domain-containing protein $1(\mathrm{SAV} 1)^{13,14}$, which 
may phosphorylate and activate the large tumor suppressor kinase $1 / 2(\mathrm{LATS} 1 / 2)^{15}$. Adapter protein MOB kinase activator $1 \mathrm{~A}(\mathrm{MOB} 1 \mathrm{~A})$ and $\mathrm{MOB} 1 \mathrm{~B}$ are also involved in the phosphorylation process ${ }^{16}$. YAP and PDZbinding motif (TAZ, also known as WW domaincontaining transcription regulator 1) are the major downstream transcription coactivators of the Hippo pathway. The phosphorylation of YAP/TAZ by the upstream kinase cascades MST1/2-LATS1/2 promotes the interaction of YAP and TAZ with cytoskeletal proteins, retains YAP and TAZ in the cytoplasm, and prevents their importation into the nucleus for transcriptional activation ${ }^{15,17}$. In contrast, when dephosphorylated, YAP can enter the nucleus and bind to the transcription factor TEA domain family member (TEADs) to control the expression of target genes ${ }^{15,18}$. YAP and TAZ rapidly shuttle between the nucleus and the cytoplasm by complex upstream components. LATS1/2mediated phosphorylation limits the rate at which YAP and TAZ are imported into the nucleus. In addition, tethering of YAP and TAZ to the cytoskeletal proteins inhibit them as cellular mechanotransduction receptors ${ }^{19}$. The NDR (nuclear Dbf2-related) protein kinase family, including NDR1/STK38 (Serine/Threonine Kinase 38) and NDR2/STK38L (Serine/Threonine Kinase 38 Like), has identified additional kinases of Hippo signaling, similar to the LATS1/2 status in the Hippo signaling pathway (Fig. 2A) ${ }^{20,21}$. It is established that the HippoYAP pathway is regulated by cell-cell contact, cell polarity, cellular mechanotransduction, and G proteincoupled receptor ligands. However, recent studies have shown that autophagy has a series of crosstalk with the Hippo-YAP pathway. In physiological settings, the two conserved pathways, autophagy and Hippo-YAP signaling are essential in the protection of homeostasis. It has been shown that the deletion of autophagy-related genes interacting with the Hippo kinase cascades is associated with an accrued propensity of laboratory animals to spontaneously develop various disorders (Table 1). In this review, we summarize the regulation of autophagy by the Hippo-YAP pathway and discuss the multidisciplinary function of Hippo-YAP-autophagy in cells and various disorders.

\section{Mechanism of autophagy}

Autophagy initiation begins with the activation of the Unc-51-like autophagy activating kinase 1 (ULK1, also known as ATG1) complex, including ULK1, ULK2, RB1 inducible coiled-coil 1 (FIP200), and ATG13. This leads to the recruitment of ATGs to the specific subcellular location called the phagophore assembly site, which activates class III phosphatidylinositol-3-kinase (PI3KC3) complex I, including VPS34, Beclin1 (mammalian homolog of yeast Atg6), p150 (mammalian homolog of yeast VPS15), and Atg14 or ultraviolet radiation resistance-associated gene protein (also known as P63), and nucleation of an annular structure of the isolation membrane, called phagophore ${ }^{22,23}$. The ATG5-ATG12 complex conjugates with ATG16 to expand the autophagosome membrane, causing the phagosome to expand into a sphere. The enzymolysis of Atg4 to LC3 (Atg8 family protein) produces cytoplasmic LC3-I, which conjugates to lipid phosphatidylethanolamine to form LC3-II and then recruits to the autophagosome membrane. Eventually, the autophagosome fuses with the lysosome and the contents are degraded, thereby enabling cellular metabolic pathways and the renewal of specific organelles (Fig. 1) ${ }^{1,23-26}$. Furthermore, p62 (also known as SQSTM1) is an autophagic modifier of the LC3 family that acts as a bridge between LC3 and ubiquitinated substrates ${ }^{27,28}$. p62-bound polyubiquitinated proteins are integrated into the completed autophagosome and degraded in autolysosomes ${ }^{29,30}$. Whereas increased p62 levels are associated with autophagy inhibition, decreased p62 levels are associated with autophagy activation, revealing that the steady-state levels of this protein could reflect the state of autophagy ${ }^{29-31}$. Thus, p62 combined with LC3 completely monitors the autophagic flux ${ }^{32}$.

\section{Crosstalk between the Hippo pathway and autophagy in Drosophila}

Hippo signaling is essential for proper growth control in Drosophila and the loss of hippo (MST1/2 in mammals) causes tissue overgrowth ${ }^{33}$. Interestingly, autophagy induction actively suppresses hippo-induced tissue overgrowth. Meanwhile, Atg1 overexpression inhibits Yorkie (YAP in mammals), further suppressing epithelial overgrowth and cell proliferation ${ }^{34}$. Mechanistically, Atg1/ ULK1 phosphorylates Yorkie at two serine residues, S74 and S97, thereby blocking transcriptional activation and inhibition of Yorkie activity. Atg1-mediated phosphorylation is an additional inhibitory input independent of the Hippo-Warts pathway ${ }^{35}$.

Steroid hormones are critical signaling molecules for growth regulation ${ }^{36}$. Warts (LATS1/2 in mammals) regulate steroid hormone production through an autophagydependent pathway (also called lipophagy). Precisely, Warts control the production of Drosophila steroid ecdysone through their effector microRNA bantam, which responds to nutrients, thus mobilizing the transport of the steroid precursor cholesterol ${ }^{37}$. Notably, YAP (mammalian Yki homolog) regulates steroidogenesis in tumor cells ${ }^{38}$, indicating that the regulation of steroidogenesis by the Wts-Yki pathway may be an evolutionarily conserved mechanism.

Trc (NDR1 in mammals) acts as a conserved regulator of autophagy and is required for early autophagosome formation in fly larvae ${ }^{39}$. Kibra, upstream components of the Drosophila Hippo pathway, act as autophagy 


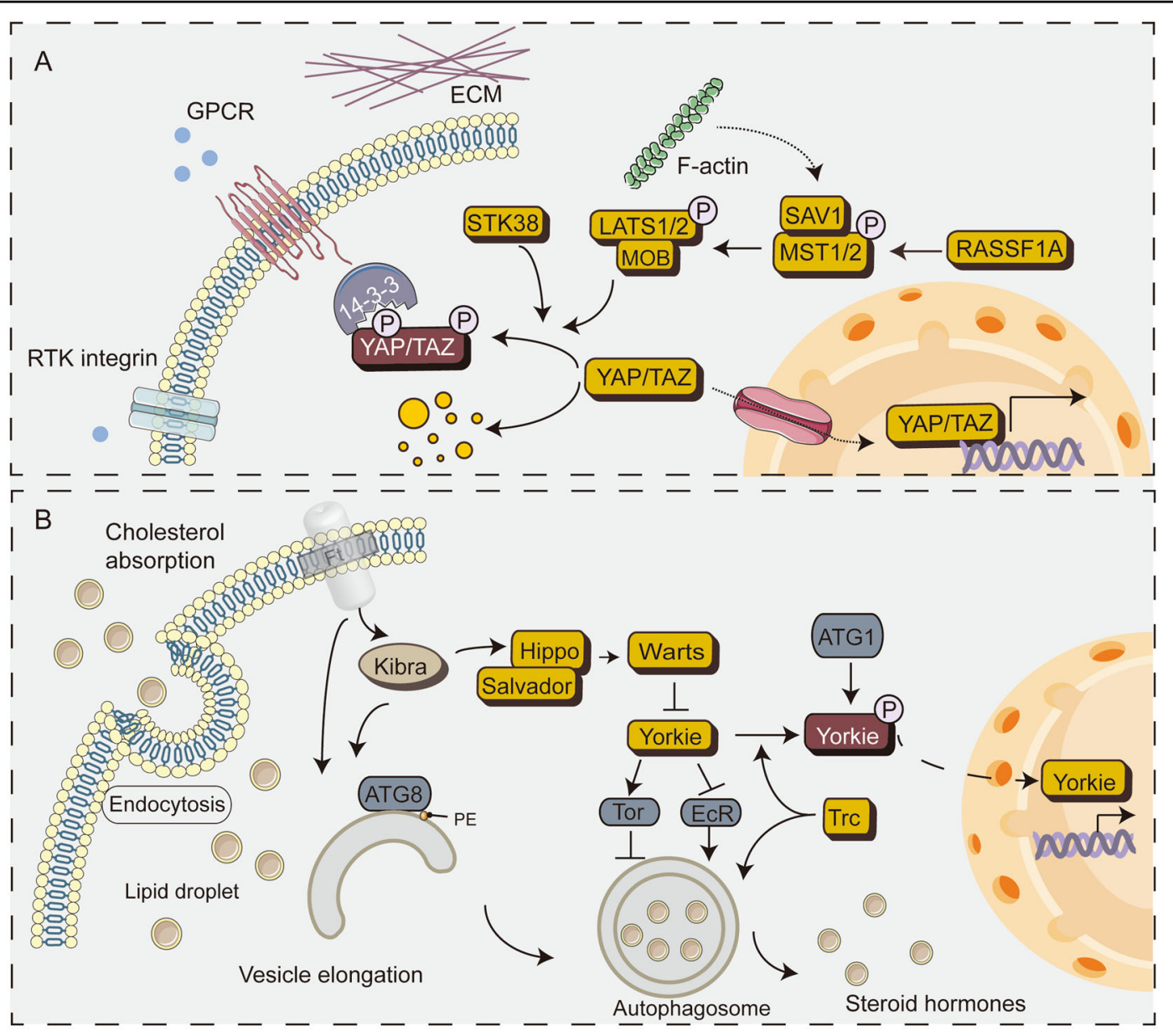

Fig. 2 Schematics diagram of the Hipoo pathway in mammals and the crosstalk between the Hippo pathway and autophagy in Drosophila. A Schematics diagram of the Hippo pathway. In mammalian cells, phosphorylation of MST1/2 activates LATS1/2, which then phosphorylates YAP/TAZ at different Ser residues. Notably, STK38 can directly phosphorylate YAP. Phosphorylated YAP/TAZ is inhibited mainly through two mechanisms: (i) cytoplasmic retention through 14-3-3 binding and (ii) proteasome degradation. Inversely, inhibition of Hippo kinase leads to nuclear accumulation of YAP/TAZ, which bind to TEADs and other transcription factors. B Schematic diagram of the crosstalk between the Hippo pathway and autophagy in Drosophila. Typically, autophagy inhibits overgrowth of epithelial tissue. When the Hippo function is reduced, this mechanism is restricted. Atg1 phosphorylates Yorkie in a Hippo-Warts-independent manner, blocks the binding of Scalloped (TEADs in mammals) and decreases the activity of Yorkie. In addition, Warts (LATS1/2 in mammals) regulates autophagy via the EcR and Tor pathways. Trc (NDR1 in mammals) promotes the formation of autophagosome. Furthermore, Kibra (WWC1/2 in mammals) and Ft (FAT1-4 in mammals) are novel autophagyregulated genes that promote ATG8-mediated elongation of the isolated membrane. The Ft mutant increases autophagy flux. The pointed and blunt arrowheads indicate activation and inhibitory interactions, respectively. Abbreviations: EcR, ecdysone receptor; Tor, target of rapamycin kinase; Trc, tricornered.

regulatory factors required for proper autophagy function $^{40}$. Drosophila protocadherin Fat $(\mathrm{Ft})$ is a cell adhesion molecule in the Hippo pathway that regulates growth and planar cell polarity ${ }^{41}$. Ft mutations cause neurodegenerative changes through autophagy defects; autophagosomes accumulate in the Ft mutant photoreceptors, which are filled with partially degraded material and damaged mitochondria ${ }^{41}$. In conclusion, the core components of the Drosophila Hippo pathway are involved in the regulation of autophagy at multiple levels and several crosstalks exist in these two conserved pathways (Fig. 2B).

\section{Regulation of autophagy by the Hippo pathway core kinase cassettes in mammals MST1/2 protein kinases}

Posttranslational modifications caused by the Hippo pathway kinases have become a powerful means to regulate autophagy in mammals. STK3/MST2 and STK4/MST1 are critical components of the Hippo pathway, which play a pivotal role in organ size control and tumor suppression. Recent studies have shown that STK3/STK4 can also be involved in the regulation of autophagy, which dynamically interacts with the Atg8 family of autophagy proteins in vitro. Specifically, they both phosphorylate LC3 at threonine 50 . 


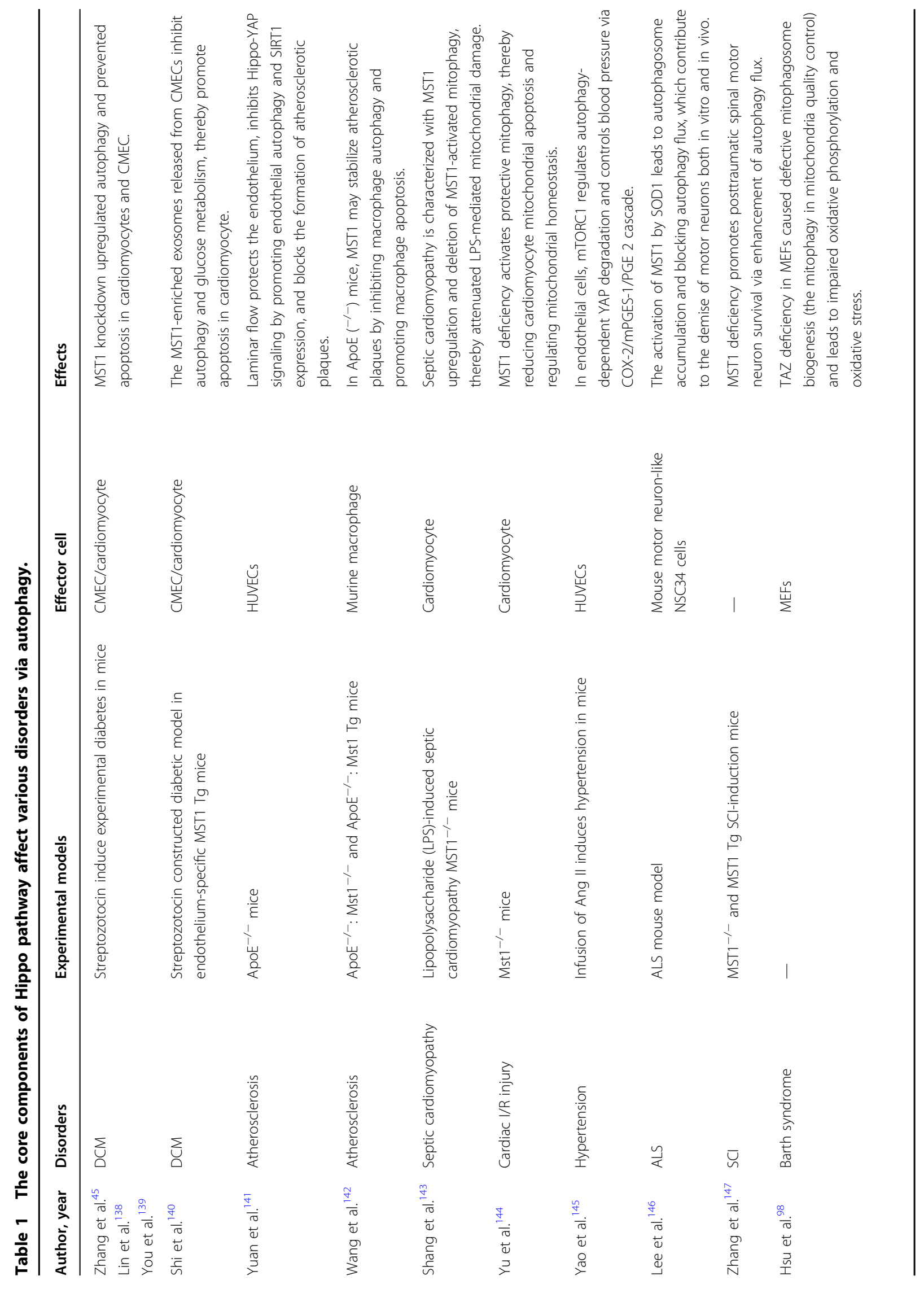




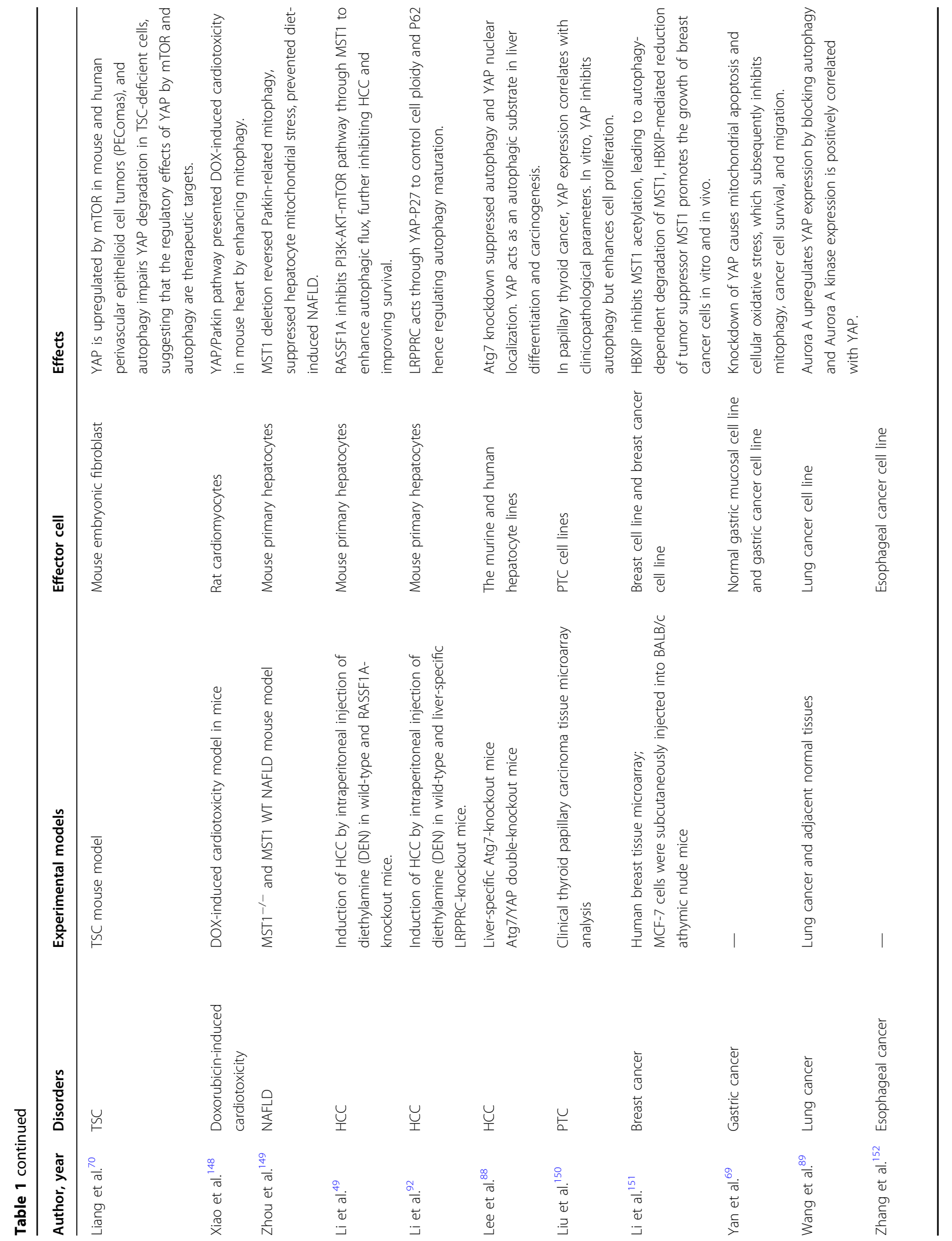


STK3/STK4-mediated phosphorylation is critical for the fusion of autophagosomes with lysosomes and the ability of cells to clear intracellular cargo (such as bacteria) ${ }^{42,43}$. STK3/ STK4 deletion leads to protein aggregate accumulation of autophagic substrates p62 and LC3-II ${ }^{42,44,45}$. STK4/MST1 phosphorylates Beclin1 in its BH3 domain at Thr108, thereby inhibiting the Beclin1-Vps34 complex, which directly inhibits autophagy. Phosphorylation cascade can enhance Beclin1-Bcl-2 interaction and induce apoptosis ${ }^{4-46}$. RASSF1A, a Hippo pathway scaffold protein, binds to MST1, promotes the activation of MST1 and causes apoptosis (induced by the death receptor signaling pathway) ${ }^{47,48}$. The loss of RASSF1A can also lead to the blockage of the autophagic flux ${ }^{49}$. The regulation of autophagy by MST1/2 is involved in several human diseases (see Table 1).

\section{NDR protein kinases}

NDR1/2 (STK38/STK38L) is regulated through alterations in the subcellular localization and phosphorylation status, which influence cell cycle, apoptosis, and autophagy in mammalian cells ${ }^{21}$. Furthermore, STK38/STK38L acts as a major stress response and plays an essential role in autophagy. Precisely, STK38 regulates itself and XPO1 (exportin-1) nuclear export by phosphorylating XPO1 on serine 1055, thereby supporting autophagy regulator Beclin1 and Hippo effector YAP shuttle into the cytoplasm $^{50}$. STK38 is also a new binding of Beclin1, which promotes autophagosome formation in mammalian cells. Conversely, STK38-depleted cells reduced PI3KC3 complex I (Beclin1-ATG14-Vps34) and PI3P formation, resulting in reduced autophagosome formation ${ }^{39}$. Moreover, STK38 regulates the chaperone-assisted selective autophagy (CASA), which initiates the CASA complex (including Hsc70, HspB8, synaptopodin-2 (SYNPO2), and the cochaperone BAG3) and mediates the degradation of misfolded, damaged, and aggregation-prone proteins ${ }^{51}$. STK38 further inhibits BAG3-mediated autophagy in a kinase activity-independent manner, which relies on the remodeling of BAG3 chaperone complexes and disrupts the interaction of $\mathrm{HspB} 8$ and $\mathrm{SYNPO}^{52}$. The underlying mechanism by which Hippo pathway core kinase cassettes regulate autophagy is shown in Fig. 3.

\section{Crosstalk between transcriptional coactivators YAP/TAZ and autophagy}

Autophagy acts as a downstream regulator of YAP/TAZ. Although YAP/TAZ controls autophagic flux by regulating the degradation of autophagosomes, YAP/TAZ is also essential for the maturation of autophagosomes into autolysosomes ${ }^{53,54}$. The use of autophagy inhibitors or endogenous knockdown of autophagy-related genes (e.g., ATG7/ 10 or $A T G 16 L 1)$ can inhibit YAP-mediated cell proliferation. Similarly, double YAP/TAZ knockdown and verteporfin (the inhibitors of YAP/TAZ ${ }^{55}$ ) treatment significantly 


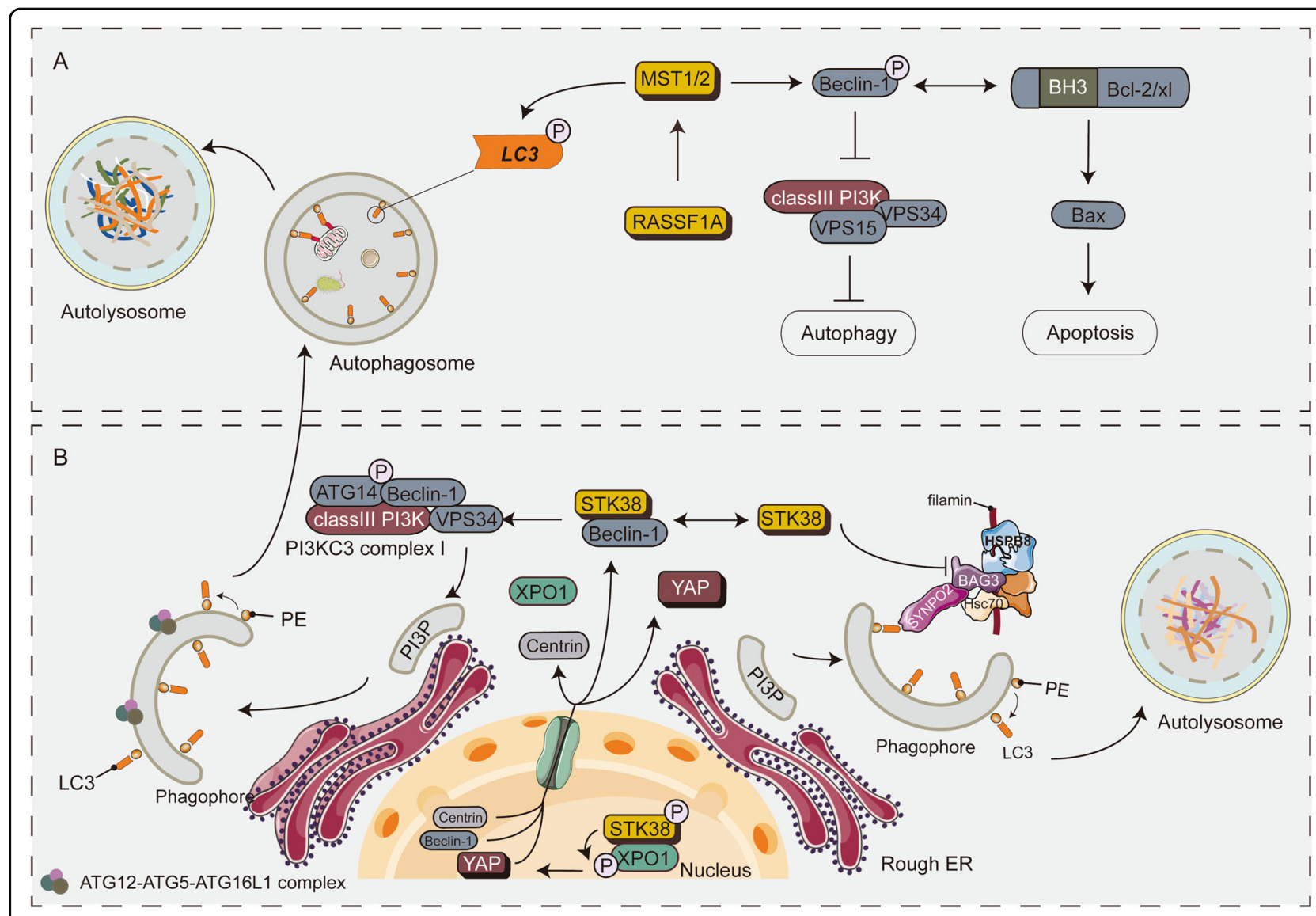

Fig. 3 A schematic diagram showing the core components of the Hippo pathway regulating autophagy in mammals. A STK3/STK4 kinases are essential for autophagy. Specifically, STK3/STK4 directly phosphorylates LC3 at threonine 50 (Thr50) in mammalian cells, promotes the fusion of autophagosomes with lysosomes and the degradation of cargo in autolysosomes. MST1/STK4 phosphorylates the BH3 domain of Beclin1 at Thr108 and inhibits Vps34 kinase activity, thereby preventing the formation of autophagosome. RASSF1A promotes the initiation and maturation of autophagy by regulating MST1. In addition, MST1 mediates the interaction between Beclin1 and Bcl-2 thereby inducing apoptosis. B STK38 phosphorylation of XPO1 on S1055 is vital for the nuclear export of crucial intracellular signal sensors such as Beclin1, YAP1, and Centrin1. Cytoplasmic STK38 interacts with Beclin1 and promotes the formation of the Beclin1-ATG14-Vps34 complex, leading to the formation of PI3P. CASA is activated in mechanically stressed cells and tissues under the regulation ofSTK38. STK38 disrupts the interaction of BAG3 with HSPB8 and SYNPO2. Moreover, CASA activation is independent of the STK38 targets BECN1.

impaired autophagy ${ }^{53,54}$. These demonstrated that the Hippo pathway maintains autophagy. In YAP/TAZ-activated cells, especially the aggressive solid tumor cells, the autophagic flux may be increased, thereby enhancing proliferation, invasion and metastasis of these cells. The underlying mechanism by which YAP/TAZ regulates autophagy is shown in Fig. 4.

\section{Contact inhibition}

Contact inhibition is a fundamental characteristic of normal cells. However, the loss of contact inhibition is an important feature of cancer cells ${ }^{56}$. The mechanical signals exerted by the physical state of cells in tissues and contact inhibition have recently been linked to the Hippo-YAP axis $^{18,57}$. The decreased proliferation and cell survival resulting from contact inhibition is partly autophagy- dependent ${ }^{54}$. The Hippo pathway serves as the functionality of YAP and TAZ by regulating their subcellular localization and protein levels ${ }^{58}$. At high cell density, YAP/TAZ is redistributed into the cytoplasm and becomes inactive ${ }^{18,59}$, failing to regulate the expression of myosin II gene. This results in a drastic reduction in the formation of the F-actin stress fibers, ultimately impairing autophagosome formation $^{54}$. Conversely, at low cell density, YAP/TAZ localizes in the nucleus and becomes active, resulting in increased F-actin formation, thus promoting autophagosome formation. In conclusion, YAP/TAZ regulates autophagy. The inhibition of YAP/TAZ function, caused by high cell density, reduces basal autophagy ${ }^{54}$. These signal crosstalks regulate the autophagy-dependent clearance of aggregation-prone proteins, survival under metabolic stress, as well as cell proliferation and differentiation ${ }^{54,60}$. 


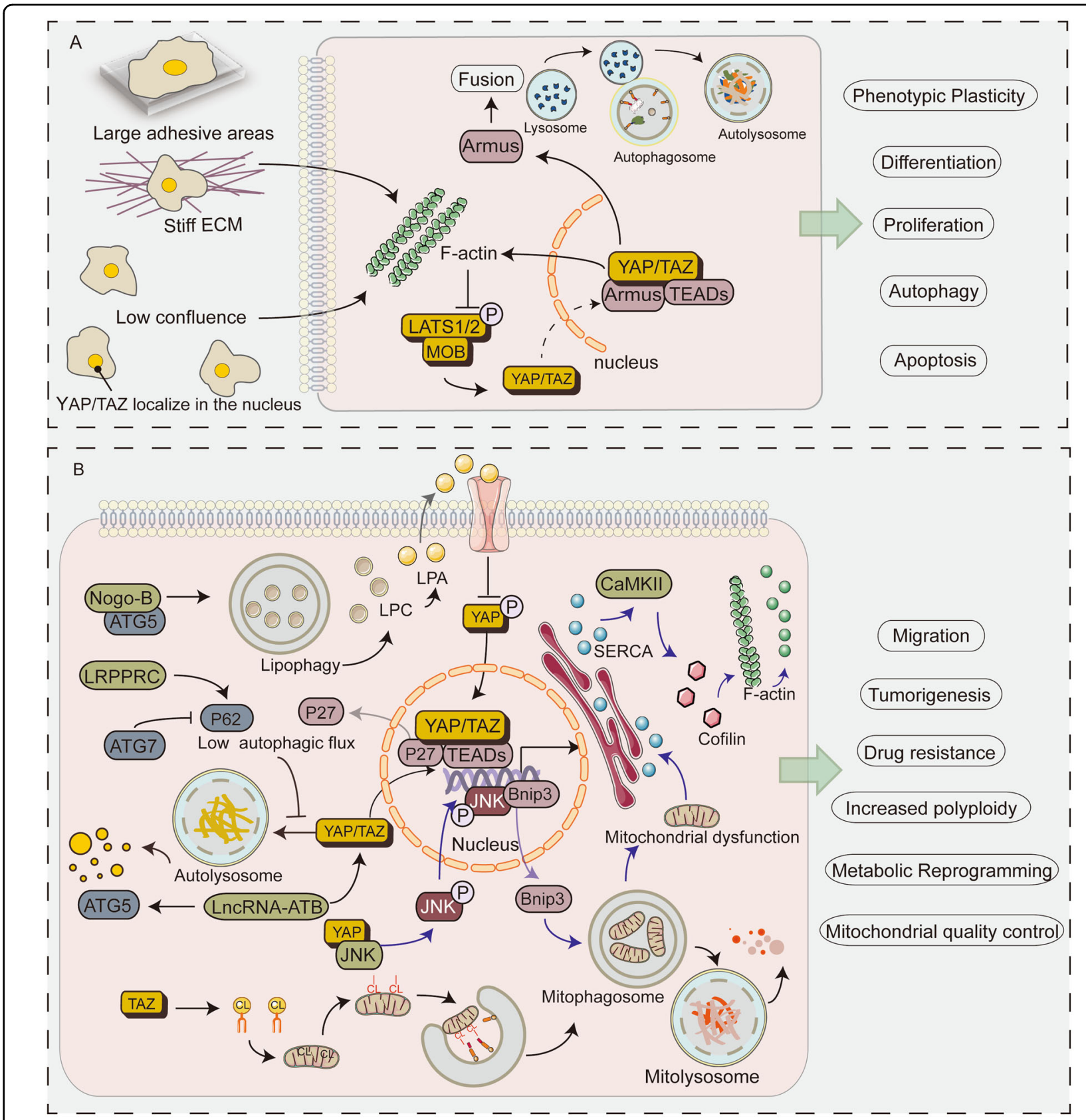

Fig. 4 Schematic diagram showing the role of YAP and TAZ in autophagy. A When cells are at low density and on a stiff extracellular matrix (ECM), Factin level is elevated leading to activation and nuclear import of YAP/TAZ, and upregulation of YAP/TAZ targets (such as myosin II and Armus). Activation of YAP/TAZ promotes F-actin accumulation. Cell mechanics control autophagic flux by regulating the transcriptional activity of YAP/TAZ. The YAP/TAZ-autophagy axis regulates a series of biological processes, such as proliferation, apoptosis, differentiation and phenotypic plasticity. B Loss of Atg7 or LRPPRC decreases autophagic flux. As an autophagic substrate, YAP cannot be degraded by autophagy, which increases nuclear localization of YAP. Activated YAP triggers accumulation of p27, which in turn leads to cellular polyploidy. IncRNA-ATB influence autophagy by participating in the transcriptional regulation of ATG5. In addition, IncRNA-ATB promotes autophagy by regulating YAP activation. Nogo-B interacts with ATG5 to promote lipophagy leading to LPC-dependent inhibition of YAP phosphorylation and enhances the oncogenic activity of YAP. YAP promotes metastasis via the mitophagy-SERCA-CaMKIl pathways and cofilin/F-actin/ lamellipodium axis. YAP binds to JNK in the cytoplasm, inducing JNK phosphorylation and nuclear localization, enhancing Bnip3 transcriptional activity. The Bnip3-induced mitophagy leads to mitochondrial dysfunction and ATP deficiency. Insufficient ATP inactivates SERCA and triggers [Ca2+] ${ }_{i}$ overload; [Ca2+] ${ }_{i}$ which phosphorylates CaMKII and inactivates coflin, ultimately leading to F-actin degradation and abrogation of lamellipodium-based migration. Cardiolipin (CL) is a phospholipid found in the inner mitochondrial membrane. TAZ is required for catalyzation of CL. When mitochondria are damaged, cardiolipin is externalized and LC3 contains CL-binding sites to initiate mitophagy, thereby maintaining mitochondrial quality control. CaMKII, Ca/calmodulin-dependent protein kinases II; CL, cardiolipin; LPA, lysophosphatidic acid; LPC, lysophosphatidylcholine; SERCA, sarco/endoplasmic reticulum Ca2+-ATPase. 


\section{Mechanotransduction}

Cells sense microenvironmental factors through a mechanotransduction system. They translate these stimuli into biochemical signals that control cell growth, differentiation, and cancer progression. Notably, YAP/TAZ is the medium for the mechanical cues indicated by the microenvironment ${ }^{18}$. YAP/TAZ is essential in the degradation of autophagosomes in both the steady-state and induced autophagy contexts. Autophagy is a downstream event of the YAP/TAZ mechanotransduction: mechanical signals act as the upstream inputs to control YAP/TAZmediated transcriptional activation of the TBC1D family members (such as Armus). This promotes the fusion of autophagosomal vesicles with lysosomes and regulates autophagy efficiency. Similar to the YAP/TAZ knockdown effect, low mechanical signal input slows down the autophagic flux ${ }^{53}$. Overall, mechanical signals can control autophagic flux through the regulation of YAP/TAZ transcriptional activity.

\section{Cancer stem cell}

Autophagy is critical in quality control, remodeling, and metabolic functions of adult and cancer stem cells $(\mathrm{CSCs})^{61}$. Similarly, YAP/TAZ regulates the biological properties of the stem cells during normal organ development and tumorigenesis ${ }^{62}$. The YAP/TAZ-autophagy connection maintains the transformed properties of the tumor cells. It also influences the acquisition of CSC status in benign cells ${ }^{53}$. Increased levels of YAP/TAZ result in the transformation of terminally differentiated epithelial cells (such as primary mammary gland cells) into stem/progenitor cells ${ }^{63}$. These reprogramming events require YAP/TAZ-dependent regulation of autophagy. YAP expression greatly increases autophagosome clearance in induced differentiated cells, whereas inactivated autophagy-related genes impair the YAP-mediated reprogramming steps ${ }^{53}$. These data suggest that YAP/ TAZ requires an effective autophagic flux to maintain CSC-inducing phenotypic plasticity.

\section{Apoptosis}

YAP overexpressed in multiple human solid tumors and inhibited apoptosis ${ }^{64,65}$. YAP located in the nucleus interacts with $\mathrm{p} 73$ and promotes apoptosis in response to DNA damage, suggesting a dual role of YAP on apopto$\mathrm{sis}^{66}$. The role of YAP in inhibiting cell apoptosis is at least partially autophagy-dependent. In ovarian and breast cancer cells, YAP knockdown increased cisplatin-induced apoptosis by decreasing autophagy ${ }^{67,68}$. Besides, YAP maintains mitophagy, the selective degradation of mitochondria by autophagy, which can block the caspase- 9 apoptotic pathway, contributing to the gastric cancer cell survival and migration ${ }^{69}$. In tuberous sclerosis complex (TSC) 1-TSC2-deficient cells, the autophagic system impairs the degradation of YAP, leading to YAP accumulation. This subsequently causes abnormal proliferation, inducing apoptosis ${ }^{70}$. The interaction between autophagy and YAP is important in the control and modulation of apoptosis and apoptotic thresholds.

\section{Metastasis}

The migration of cancer cells into the circulatory or lymphatic system to form metastases is an extremely complex process in which the Hippo-YAP-autophagy axis is extensively involved ${ }^{71-73}$. Recent functional studies suggest that YAP mediates cancer metastasis via the modulation of actin dynamics ${ }^{74,75}$ and the control of transcriptional activity ${ }^{76,77}$, and along with the long noncoding RNA (lncRNA)-dependent manner ${ }^{78,79}$. Once the cancer cells spread to the systemic circulation and colonize distant organs, autophagic flux is induced to respond to the stressful microenvironments, including hypoxia, nutritional deficiencies and the extracellular matrix detachment ${ }^{80,81}$. F-actin polymerization drives the cellular membrane extension in lamellipodia, leading to cytoskeletal rearrangement, thus promoting migration ${ }^{74,82}$. YAP deficiency promoted the phosphorylation of JNK (c-Jun $\mathrm{N}$-terminal kinases), which activated Bnip3 transcriptional activity and contributed to the Bnip3-required mitophagy. Higher Bnip3 caused mitochondrial dysfunction and ATP shortage, degraded F-actin via SERCA/[Ca2 + ] i/CaMKII/cofilin axis, and attenuated lamellipodiumbased migration ${ }^{75}$. In the triple-negative breast cancer (TNBC) cells, autophagy promoted YAP nuclear localization, promoting TNBC cell migration and invasion ${ }^{83}$.

\section{Hepatocarcinogenesis}

YAP and TAZ are widely activated in human malignancies, which plays a vital role in tumorigenesis and the growth of most solid tumors ${ }^{84}$. For example, overexpression of YAP causes prominent hepatomegaly and induces tumor stem cell attributes, and hepatocarcinogenesis $^{85,86}$. Autophagy maintains hepatic organ size and differentiation and, when autophagy is impaired, YAP is a driver of tissue remodeling and tumorigenesis ${ }^{87}$. In vivo, the liver-specific Atg7-deletion (Atg7 knockout (KO)) mice showed an 8.5-fold increase in the relative liver weight compared to the control mice at three months. Dysplastic nodules appeared at 8 months, whereas hepatocellular carcinoma (HCC) developed at 12 months. Meanwhile, the Atg7/YAP double KO mice attenuated hepatomegaly and hepatocarcinogenesis with significantly lower tumor size and number than the Atg7-KO mice ${ }^{88}$.

As expected, knockdown of Atg7 or Atg5 reduced autophagic flux. Interestingly, shAtg7 or shAtg5 induced nuclear translocation of YAP leading to the activation of TEAD4. Furthermore, YAP colocalized with autophagosomes, so that the cytoplasmic degradation of YAP was at 
least partially autophagy-dependent ${ }^{88,89}$. As YAP is an essential downstream mediator of tissue remodeling, progenitor cell activation, tumorigenesis, and drug resistance in the autophagy-deficient liver, the concomitant loss of YAP attenuates these abnormalities ${ }^{88,90}$. LRPPRC is a mitochondrion-associated protei $^{91}$. The loss of LRPPRC expression promotes hepatocarcinogenesis ${ }^{92}$. Specifically, the deletion of LRPPRC leads to liver-specific YAP nuclear accumulation and induces accumulation of the cyclin-dependent kinase inhibitor p27, which in turn leads to cell polyploidy ${ }^{92,93}$. Concurrently, the deletion of mitochondrion-associated protein LRPPRC reduces $\mathrm{p} 62$ protein levels and impairs autophagic maturation. In vitro, LRPPRC knockdown synergistically enhances the diethylamine-induced genomic instability and hepatocarcinogenesis $^{92}$. The lncRNAs are associated with clinicopathological parameters of HCC and can be used as biomarkers for HCC diagnosis ${ }^{94}$. Precisely, lncRNA-ATB activates the YAP-dependent autophagy and increases the expression of ATG5. The high expression of IncRNAATB is associated with poor prognosis and pathological characteristics of $\mathrm{HCC}^{95}$. Nogo-B, an endoplasmic reticulum residential protein, is highly expressed and promotes tumorigenesis in HCC. Mechanistically, Nogo-B interacts with ATG5 to encourage droplet lipid degradation and induces lipophagy-mediated oxidized lowdensity lipoprotein metabolism and subsequent lysophosphatidic acid-stimulated YAP oncogenic activity ${ }^{96}$.

\section{Mitochondrial quality control}

Mitophagy removes damaged mitochondria through autophagy, which is essential for mitochondrial quality control, metabolic homeostasis, and energy supply ${ }^{97}$. Notably, TAZ is required for mitophagy but not autophagosome biogenesis ${ }^{98}$. TAZ is a phospholipid transacylase that catalyzes the remodeling of cardiolipin, a mitochondrial endosomal phospholipid. The redistribution of cardiolipin controls the initiation of mitophagy $^{98,99}$. Mechanistically, TAZ knockdown and inducible TAZ depletion prevent LC3 vesicles from recognizing mitophagosomes, thereby inhibiting mitophagy initiation. This leads to impaired oxidative phosphorylation and oxidative stress. Thus, TAZ is required for the initiation of mitophagy. It is involved in mitochondrial quality control. Mutations of the TAZ gene can cause Barth syndrome ${ }^{98,100}$.

\section{Hippo-YAP-autophagy axis in clinical applications}

Autophagy is an attractive therapeutic target in numerous diseases. As autophagy has a wide correlation with normal homeostasis, targeting it is particularly challenging. In contrast, the role of the Hippo pathway in cancer is widely described. It plays a vital role in tissue renewal and repair. Therefore, targeting the Hippo-YAP-autophagy axis might provide several promising targets. The emerging link between the Hippo pathway and autophagy is now largely implicated in pathophysiological processes, such as cancer, metabolic and neurodegenerative diseases, and cardiovascular diseases (Fig. 5) ${ }^{101-103}$. Here we introduce some small molecules or drugs that target the Hippo core components autophagy regulatory network (Table 2).

\section{Cancer}

Autophagy is involved in several tumor progression stages, including tumorigenesis, progression, and malignant status maintenance ${ }^{73}$. In the early stages of tumorigenesis, autophagy maintains genome stability by removing oncogenic protein substrates, toxic unfolded proteins, and damaged organelles. This prevents chronic tissue damage, cell damage, and inflammation. Moreover, autophagy inhibits the accumulation of carcinogenic p62 protein aggregates, thereby promoting tumor suppres$\operatorname{sion}^{104-106}$. At an advanced tumor stage, the autophagic flux increases to cope with various environmental pressures, including hypoxia, nutritional deficiencies, DNA damage, metabolic stress, and chemotherapy. This maintains the survival and growth of tumor cells, and promotes tumor invasion and metastasis ${ }^{9,73,107}$. However, when blocking or promoting autophagy at different stages of tumor development, the biological effects on the tumor cell behavior may vary.

As previously mentioned, YAP acts as an autophagic substrate. The expression of YAP protein and YAP target genes is regulated by the autophagic flux ${ }^{88,89}$. Thus, some small molecules that induce autophagy can reduce the oncogenic activity of YAP/TAZ. Curcumin, a natural polyphenolic compound ${ }^{108}$, induces autophagy in colon cancer cells, further inhibiting cell proliferation and YAP expression ${ }^{109}$. Silibinin, a flavonolignan from the seeds of milk thistle, induced glioblastoma cell apoptosis and autophagy via inhibition of mammalian target of rapamycin and $\mathrm{YAP}^{110}$. Shikonin is the main bioactive ingredient extracted from the root of Lithospermum erythrorhizon ${ }^{111}$, which exerts anti-colon cancer effects similar to silibinin and inhibits YAP activity by inducing autophagy ${ }^{112}$.

As of May 2020, a search for "autophagy and cancer" on ClinicalTrials.gov revealed 72 studies focusing on the inhibition and evaluation of autophagy to improve the clinical prognosis for cancer patients. Targeted drugs either as single agents or in combinations can exert antitumor effects by enhancing both apoptotic and toxic autophagic processes $^{113}$. For instance, neratinib (ERBB1/2/4 inhibitor) enhanced [pazopanib (the kinase inhibitor) + entinostat (histone deacetylase inhibitor)] lethality against sarcoma and other tumor cell types in vitro and in vivo. Specifically, the triplet combination increases the phosphorylation of YAP/TAZ and promotes the conversion of LC3 and expression of Beclin1 and ATG13, which together enhance 


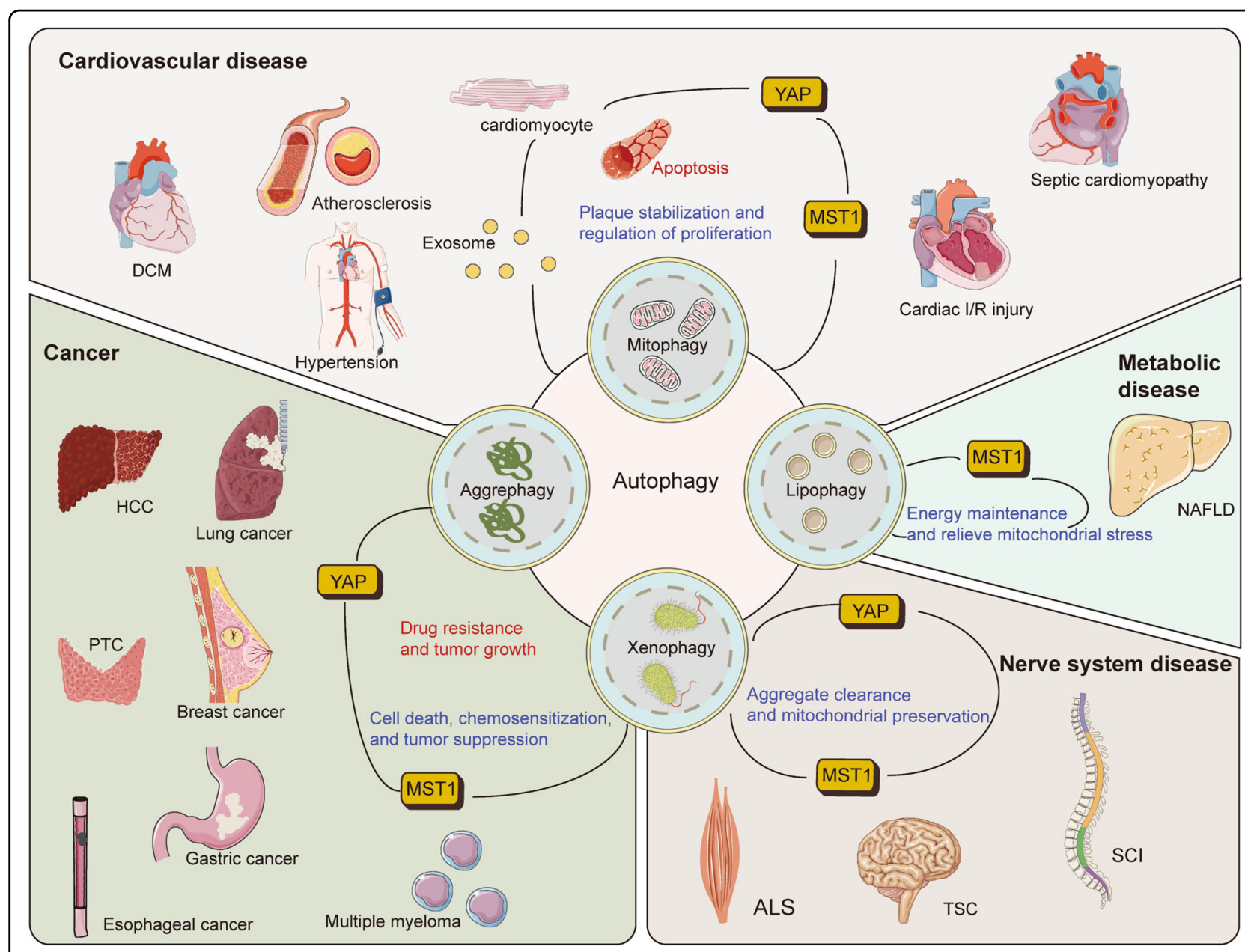

Fig. 5 The core components of hippo pathway affect various disorders via autophagy. Hippo-YAP axis regulates autophagy and affects the development of disease progression. Inhibition of disease progression (beneficial process) is shown in blue, whereas promotion of disease progression (harmful process) is shown in red. In many diseases, autophagy clears dysfunctional mitochondria and protein aggregates. As two conserved signaling pathways, the Hippo pathway and autophagy intersect in the regulation of cell death and proliferation, tumorigenesis, and survival and growth of tumor cells. ALS, amyotrophic lateral sclerosis; DCM, diabetic cardiomyopathy; HCC, hepatocellular carcinoma; I/R, ischemiareperfusion; NAFLD, non-alcoholic fatty liver disease; PTC, papillary thyroid carcinoma; SCl, spinal cord injury; TSC, tuberous sclerosis complex.

autophagosome formation $^{114,115}$. The mammalian STK 26/ MST4 stimulates ATG4B activity and increases autophagic flux by phosphorylating ATG4B ${ }^{116}$. The MST4-MOB4 complex can disrupt the assembly of the MST1-MOB1 complex by alternative pairing, thereby increasing YAP activity $^{117}$. Neratinib degrades MST4 via autophagy, enhancing LATS1/2 phosphorylation, and is also required for YAP/TAZ inactivation ${ }^{118}$

Typically, although LATS1 plays a tumor suppressor role in the Hippo pathway, it also exerts a pro-survival function in the HCC cells ${ }^{119}$. Sorafenib (Srf), a multi-kinase inhibitor that promotes autophagy, is the standard treatment for advanced $\mathrm{HCC}^{120,121}$. The blockade of LATS1 expression resulted in increased Srf-induced apoptosis and decreased cell viability in vitro, as well as reduced tumor growth in vivo. LATS1 promotes K27-ubiquitination of Beclin1 on lysines K32 and K263, which inhibits autophagy induction and autophagic flux in HCC cells after Srf treatment. In Srfnonrespondent patient, LATS1 expression is significantly increased, suggesting that LATS1 is a clinically relevant biomarker for Srf sensitivity ${ }^{119}$. The revelation of LATS1 functionally independent of the kinase activity in autophagy regulation requires consideration for targeted LATS1 kinases therapy.

\section{Non-cancerous diseases}

Autophagy plays a pivotal role in protein quality control, especially in maintaining metabolic homeostasis ${ }^{122}$. Dihydrotanshinone I, a natural monomeric compound isolated from Salvia miltiorrhiza Bunge, can improve liver function and reduce liver fibrosis. The underlying mechanism is associated with the cytoplasmic retention of 
Table 2 Small molecules or drugs that target the Hippo core components autophagy regulatory network.

\begin{tabular}{|c|c|c|c|c|c|}
\hline Organ & & Diseases & Small molecules or drugs & Effects & Reference \\
\hline & \multirow[t]{2}{*}{ Brain } & $\mathrm{SAH}$ & Melatonin & $\begin{array}{l}\text { Melatonin play a neuroprotective role by regulating } \\
\text { the homeostasis between apoptosis and autophagy } \\
\text { through the oxygen species (ROS)-MST1 pathway }\end{array}$ & Shi et al. ${ }^{134}$ \\
\hline & & Glioblastoma & Silibinin & $\begin{array}{l}\text { Silibinin induced glioblastoma cell apoptosis and } \\
\text { autophagy via inhibition of mTOR and YAP. }\end{array}$ & Bai et al. ${ }^{110}$ \\
\hline & \multirow[t]{2}{*}{ Heart } & DCM & Melatonin & $\begin{array}{l}\text { Melatonin protects against DCM by increasing } \\
\text { autophagy and reducing apoptosis through MST1/ } \\
\text { Sirt3 signaling }\end{array}$ & Zhang et al. ${ }^{131}$ \\
\hline & & Cardiotoxicity & Adriamycin & $\begin{array}{l}\text { HMGB1 is functionally related to YAP and } \\
\text { participates in adriamycin-induced cardiotoxicity by } \\
\text { upregulating autophagy. }\end{array}$ & Luo et al. ${ }^{129}$ \\
\hline & \multirow[t]{2}{*}{ Liver } & $\mathrm{HCC}$ & Sorafenib & $\begin{array}{l}\text { Sorafenib promotes autophagy and is the standard } \\
\text { treatment for advanced HCC, LATS1 restricts lethal } \\
\text { autophagy in sorafenib-induced HCC cells. }\end{array}$ & Tang et al. ${ }^{119}$ \\
\hline & & Hepatic fibrosis & Dihydrotanshinone I & $\begin{array}{l}\text { Dihydromorphone I exerts anti-fibrotic effects by } \\
\text { blocking the YAP-TEAD2 complex and stimulating } \\
\text { autophagy }\end{array}$ & Ge et al. ${ }^{123}$ \\
\hline & Pancreas & Pancreatic cancer & Neratinib & $\begin{array}{l}\text { Neratinib degrades MST4 via autophagy and is } \\
\text { essential for the inactivation of YAP/TAZ. }\end{array}$ & Dent et al. ${ }^{118}$ \\
\hline & \multirow[t]{2}{*}{ Colon } & Colon cancer & Curcumin & Curcumin induces autophagy via inhibition of YAP. & Zhu et al. ${ }^{109}$ \\
\hline & & Colon cancer & Shikonin & $\begin{array}{l}\text { Shikonin effectively suppress colon cancer cell } \\
\text { viability and migration, and induces autophagy via } \\
\text { inhibiting the activity of YAP. }\end{array}$ & Zhu et al. ${ }^{112}$ \\
\hline
\end{tabular}

DCM diabetic cardiomyopathy, HCC hepatocellular carcinoma, SAH subarachnoid hemorrhage.

YAP, thereby causing downregulation of fibrogenic gene expression, which stimulates autophagic flux and accelerates the degradation of the liver collagen ${ }^{123}$.

Autophagy offers promising targets for the prevention and treatment of cardiovascular diseases ${ }^{122}$. It has been reported that HMGB1, a chromosomal protein, acts as an autophagy sensor and induces autophagy after prolonged cellular stress $^{124,125}$. The expression of HMGB1 is highly correlated with YAP activity, which is involved in tumorigenesis and acquisition of the tumor stem cell characteristics ${ }^{126,127}$. Adriamycin, an anthracycline chemotherapy drug, can also cause cardiotoxicity ${ }^{128}$. Specifically, adriamycin upregulates HMGB1 expression and induces cardiomyocyte autophagy followed by cardiac damage, whereas YAP reverses adriamycin-induced cardiac damage by downregulating HMGB1 $^{129}$. Melatonin regulates autophagy and has both chronobiotic and cytoprotective properties ${ }^{130}$. Melatonin significantly alleviates left ventricle remodeling and cardiac dysfunction in dilated cardiomyopathy by inducing autophagy and alleviating mitochondrial dysfunction, which is partially dependent on MST1/Sirt3 signaling ${ }^{131,132}$.

Autophagy is essential for maintaining proteostasis and a healthy mitochondrial pool, especially in maintaining the homeostasis of non-dividing nerve cells ${ }^{133}$. Melatonin plays a cytoprotective role in a variety of neurodegenerative diseases ${ }^{130}$. In subarachnoid hemorrhage-induced rats, melatonin can regulate the homeostasis between apoptosis and autophagy by inhibiting the ROS-MST1 pathway $^{134}$.

\section{Conclusions and perspectives}

The understanding of the regulatory network between the Hippo-YAP pathway and autophagy has gradually been enriched in recent years. In this review, we show that these two highly conserved signaling pathways are widely involved in pathophysiological processes such as apoptosis, cell proliferation, cell differentiation, and metabolism, and can influence the pathogenesis of human diseases. This multidisciplinary view improves our understanding of why these two signaling pathways have been preserved throughout evolution. However, variations in the activity between autophagy and the Hippo-YAP pathway in different tissue types, tumor microenvironments, and disease states are some of the fundamental puzzles yet to be resolved. In addition, the paradoxical effect of autophagy in cancer makes autophagy-targeted therapy in cancer 
controversial. However, the Hippo pathway dysregulation occurs in a wide range of human cancers. This is essential in the development of novel and more specific drugs. For example, a combination of Hippo pathway-targeted drugs with autophagy inhibitors and inducers may be potential therapies for various human diseases. A better understanding and targeting of the Hippo-YAP-autophagy axis is an auspicious direction.

\section{Acknowledgements}

Dr. Tianmin Xu was supported by funding from the Department of Science and Technology of Jilin Province (grant number 20180201032YY and 20200602008ZP); the Department of Finance of Jilin Province (grant number 2019SCZT017)

\section{Conflict of interest}

The authors declare that they have no conflict of interest.

\section{Publisher's note}

Springer Nature remains neutral with regard to jurisdictional claims in published maps and institutional affiliations.

Received: 11 June 2020 Revised: 7 August 2020 Accepted: 21 August 2020 Published online: 20 October 2020

\section{References}

1. Mizushima, N. \& Komatsu, M. Autophagy: renovation of cells and tissues. Cell 147, 728-741 (2011)

2. Levine, B. \& Kroemer, G. Autophagy in the pathogenesis of disease. Cell 132, 27-42 (2008).

3. Rabinowitz, J. D. \& White, E. Autophagy and metabolism. Science $\mathbf{3 3 0}$, 1344-1348 (2010).

4. Tsukada, M. \& Ohsumi, Y. Isolation and characterization of autophagydefective mutants of Saccharomyces cerevisiae. FEBS Lett. 333, 169-174 (1993).

5. Ktistakis, N. T. \& Tooze, S. A. Digesting the expanding mechanisms of autophagy. Trends Cell Biol. 26, 624-635 (2016).

6. Mizushima, N., Yoshimori, T. \& Ohsumi, Y. The role of Atg proteins in autophagosome formation. Annu. Rev. Cell Dev. Biol. 27, 107-132 (2011).

7. Choi, A. M. K., Ryter, S. W. \& Levine, B. Autophagy in human health and disease. N. Engl. J. Med. 368, 651-662 (2013).

8. Kocaturk, N. M. et al. Autophagy as a molecular target for cancer treatment. Eur. J. Pharm. Sci. 134, 116-137 (2019).

9. White, E. Deconvoluting the context-dependent role for autophagy in cancer. Nat. Rev. Cancer 12, 401-410 (2012).

10. Chen, H.-Y. \& White, E. Role of autophagy in cancer prevention. Cancer Prev. Res. 4, 973-983 (2011).

11. Amaravadi, R., Kimmelman, A. C. \& White, E. Recent insights into the function of autophagy in cancer. Genes Dev. 30, 1913-1930 (2016).

12. Piccolo, S., Dupont, S. \& Cordenonsi, M. The biology of YAP/TAZ: hippo signaling and beyond. Physiol. Rev. 94, 1287-1312 (2014).

13. Udan, R. S., Kango-Singh, M., Nolo, R., Tao, C. \& Halder, G. Hippo promotes proliferation arrest and apoptosis in the Salvador/Warts pathway. Nat. Cell Biol. 5, 914-920 (2003).

14. Kango-Singh, M. et al. Shar-pei mediates cell proliferation arrest during imaginal disc growth in Drosophila. Development 129, 5719-5730 (2002).

15. Hao, Y., Chun, A., Cheung, K., Rashidi, B. \& Yang, X. Tumor suppressor LATS1 is a negative regulator of oncogene YAP. J. Biol. Chem. 283 5496-5509 (2008)

16. Xiong, S. et al. Regulation of protein interactions by Mps one binder (MOB1) phosphorylation. Mol. Cell. Proteomics 16, 1111-1125 (2017).

17. Moroishi, T. et al. The Hippo pathway kinases LATS1/2 suppress cancer immunity. Cell 167, https://doi.org/10.1016/j.cell.2016.11.005 (2016).

18. Dupont, S. et al. Role of YAP/TAZ in mechanotransduction. Nature $\mathbf{4 7 4}$ 179-183 (2011).
19. Panciera, T., Azzolin, L., Cordenonsi, M. \& Piccolo, S. Mechanobiology of YAP and TAZ in physiology and disease. Nat. Rev. Mol. Cell Biol. 18, 758-770 (2017).

20. Sharif, A. A. D. \& Hergovich, A. The NDR/LATS protein kinases in immunology and cancer biology. Semin. Cancer Biol. 48, 104-114 (2018).

21. Hergovich, A. The roles of NDR protein kinases in Hippo signalling. Genes $\mathbf{7}$ https://doi.org/10.3390/genes7050021 (2016).

22. Liang, $X$. H. et al. Induction of autophagy and inhibition of tumorigenesis by beclin 1. Nature 402, 672-676 (1999).

23. Dikic, I. \& Elazar, Z. Mechanism and medical implications of mammalian autophagy. Nat. Rev. Mol. Cell Biol. 19, 349-364 (2018).

24. Abada, A. \& Elazar, Z. Getting ready for building: signaling and autophagosome biogenesis. EMBO Rep. 15, 839-852 (2014).

25. Lamb, C. A., Yoshimori, T. \& Tooze, S. A. The autophagosome: origins unknown, biogenesis complex. Nat. Rev. Mol. Cell Biol. 14, 759-774 (2013).

26. Klionsky, D. J. et al. Guidelines for the use and interpretation of assays for monitoring autophagy (3rd edition). Autophagy 12, https://doi.org/10.1080/ 15548627.2015.1100356 (2016).

27. Jiang, P. \& Mizushima, N. LC3- and p62-based biochemical methods for the analysis of autophagy progression in mammalian cells. Methods 75, 13-18 (2015).

28. Bjørkøy, G. et al. p62/SQSTM1 forms protein aggregates degraded by autophagy and has a protective effect on huntingtin-induced cell death. J. Cell Biol. 171, 603-614 (2005).

29. Bartlett, B. J. et al. p62, Ref(2)P and ubiquitinated proteins are conserved markers of neuronal aging, aggregate formation and progressive autophagic defects. Autophagy 7, 572-583 (2011).

30. Nezis, I. P. et al. Ref(2)P, the Drosophila melanogaster homologue of mammalian p62, is required for the formation of protein aggregates in adult brain. J. Cell Biol. 180, 1065-1071 (2008).

31. Lee, J.-H. et al. Lysosomal proteolysis and autophagy require presenilin 1 and are disrupted by Alzheimer-related PS1 mutations. Cell 141, 1146-1158 (2010)

32. Klionsky, D. J. et al. Guidelines for the use and interpretation of assays for monitoring autophagy. Autophagy 8, 445-544 (2012).

33. Moya, I. M. \& Halder, G. Hippo-YAP/TAZ signalling in organ regeneration and regenerative medicine. Nat. Rev. Mol. Cell Biol. 20, 211-226 (2019).

34. Pérez, E., Das, G., Bergmann, A. \& Baehrecke, E. H. Autophagy regulates tissue overgrowth in a context-dependent manner. Oncogene 34, 3369-3376 (2015).

35. Tyra, L. K., Nandi, N., Tracy, C. \& Krämer, H. Yorkie growth-promoting activity is limited by Atg1-mediated phosphorylation. Dev. Cell 52, https://doi.org/ 10.1016/j.devcel.2020.01.011 (2020).

36. Danielsen, E. T. et al. A Drosophila genome-wide screen identifies regulators of steroid hormone production and developmental timing. Developmental cell 37, 558-570 (2016)

37. Texada, M. J. et al. Autophagy-mediated cholesterol trafficking controls steroid production. Dev. Cell 48, https://doi.org/10.1016/j.devcel.2019.01.007 (2019).

38. Farrow, J. M., Yang, J. C. \& Evans, C. P. Autophagy as a modulator and target in prostate cancer. Nat. Rev. Urol. 11, 508-516 (2014).

39. Joffre, C. et al. The pro-apoptotic STK38 kinase is a new Beclin1 partner positively regulating autophagy. Curr. Biol. 25, 2479-2492 (2015).

40. Jin, A., Neufeld, T. P. \& Choe, J. Kibra and aPKC regulate starvation-induced autophagy in Drosophila. Biochem. Biophys. Res. Commun. 468, 1-7 (2015).

41. Napoletano, F. et al. Polyglutamine Atrophin provokes neurodegeneration in Drosophila by repressing fat. EMBO J. 30, 945-958 (2011).

42. Wilkinson, D. S. et al. Phosphorylation of LC3 by the Hippo kinases STK3/STK4 is essential for autophagy. Mol. Cell 57, 55-68 (2015).

43. Shrestha, B. K. et al. NIMA-related kinase 9-mediated phosphorylation of the microtubule-associated LC3B protein at Thr-50 suppresses selective autophagy of p62/sequestosome 1. J. Biol. Chem. 295, 1240-1260 (2020).

44. Maejima, Y. et al. Mst1 inhibits autophagy by promoting the interaction between Beclin1 and Bcl-2. Nat. Med. 19, 1478-1488 (2013).

45. Zhang, M. et al. MST1 coordinately regulates autophagy and apoptosis in diabetic cardiomyopathy in mice. Diabetologia 59, 2435-2447 (2016).

46. Lee, E. F. et al. Structural insights into BCL2 pro-survival protein interactions with the key autophagy regulator BECN1 following phosphorylation by STK4/MST1. Autophagy 15, 785-795 (2019).

47. Pefani, D.-E. et al. TGF- $\beta$ targets the Hippo pathway scaffold RASSF1A to facilitate YAP/SMAD2 nuclear translocation. Mol. Cell 63, 156-166 (2016). 
48. Oh, H. J. et al. Role of the tumor suppressor RASSF1A in Mst1-mediated apoptosis. Cancer Res. 66, 2562-2569 (2006).

49. Li, W. et al. Suppressor of hepatocellular carcinoma RASSF1A activates autophagy initiation and maturation. Cell Death Differ. 26, 1379-1395 (2019).

50. Martin, A. P. et al. STK38 kinase acts as XPO1 gatekeeper regulating the nuclear export of autophagy proteins and other cargoes. EMBO Rep. 20, e48150 (2019).

51. Ulbricht, A. et al. Cellular mechanotransduction relies on tension-induced and chaperone-assisted autophagy. Curr. Biol. 23, 430-435 (2013).

52. Klimek, C. et al. The Hippo network kinase STK38 contributes to protein homeostasis by inhibiting BAG3-mediated autophagy. Biochim. Biophys. Acta Mol. Cell Res. 1866, 1556-1566 (2019).

53. Totaro, A. et al. Cell phenotypic plasticity requires autophagic flux driven by YAP/TAZ mechanotransduction. Proc. Natl Acad. Sci. USA 116, 17848-17857 (2019).

54. Pavel, M. et al. Contact inhibition controls cell survival and proliferation via YAP/TAZ-autophagy axis. Nat. Commun. 9, 2961 (2018).

55. Wang, C. et al. Verteporfin inhibits YAP function through up-regulating 14-33sigma sequestering YAP in the cytoplasm. Am. J. Cancer Res. 6, 27-37 (2016).

56. Holley, R. W. Control of growth of mammalian cells in cell culture. Nature 258, 487-490 (1975).

57. Zhao, B. et al. Inactivation of YAP oncoprotein by the Hippo pathway is involved in cell contact inhibition and tissue growth control. Genes Dev. 21, 2747-2761 (2007).

58. Hong, W. \& Guan, K. L. The YAP and TAZ transcription co-activators: key downstream effectors of the mammalian Hippo pathway. Semin. Cell Dev. Biol. 23, 785-793 (2012).

59. Aragona, M. et al. A mechanical checkpoint controls multicellular growth through YAP/TAZ regulation by actin-processing factors. Cell 154, 1047-1059 (2013).

60. Gumbiner, B. M. \& Kim, N.-G. The Hippo-YAP signaling pathway and contact inhibition of growth. J. Cell Sci. 127, 709-717 (2014).

61. Boya, P., Codogno, P. \& Rodriguez-Muela, N. Autophagy in stem cells: repair, remodelling and metabolic reprogramming. Development 145, https:/doi. org/10.1242/dev.146506 (2018).

62. Park, J. H., Shin, J. E. \& Park, H. W. The role of Hippo pathway in cancer stem cell biology. Mol. Cell. 41, 83-92 (2018).

63. Panciera, T. et al. Induction of expandable tissue-specific stem/progenitor cells through transient expression of YAP/TAZ. Cell Stem Cell 19, 725-737 (2016).

64. Huang, J., Wu, S., Barrera, J., Matthews, K. \& Pan, D. The Hippo signaling pathway coordinately regulates cell proliferation and apoptosis by inactivating Yorkie, the Drosophila homolog of YAP. Cell 122, 421-434 (2005).

65. Furth, N., Aylon, Y. \& Oren, M. p53 shades of Hippo. Cell Death Differ. 25, 81-92 (2018)

66. Lapi, E. et al. PML, YAP, and p73 are components of a proapoptotic autoregulatory feedback loop. Mol. Cell 32, 803-814 (2008).

67. Xiao, L. et al. YAP induces cisplatin resistance through activation of autophagy in human ovarian carcinoma cells. OncoTargets Ther. 9, 1105-1114 (2016).

68. Jiang, Y. et al. Cisplatin-induced autophagy protects breast cancer cells from apoptosis by regulating yes-associated protein. Oncol. Rep. 38, 3668-3676 (2017).

69. Yan, H. et al. Yap regulates gastric cancer survival and migration via SIRT1/ Mfn2/mitophagy. Oncol. Rep. 39, 1671-1681 (2018).

70. Liang, N. et al. Regulation of YAP by mTOR and autophagy reveals a therapeutic target of tuberous sclerosis complex. J. Exp. Med. 211, 2249-2263 (2014).

71. Lee, C.-K. et al. Tumor metastasis to lymph nodes requires YAP-dependent metabolic adaptation. Science 363, 644-649 (2019).

72. Zhang, Z. et al. OTUB2 promotes cancer metastasis via Hippo-independent activation of YAP and TAZ. Mol. Cell 73, https://doi.org/10.1016/j. molcel.2018.10.030 (2019)

73. $L i, X ., H e, S . \& M a, B$. Autophagy and autophagy-related proteins in cancer. Mol. Cancer 19, 12 (2020).

74. Qiao, Y. et al. YAP regulates actin dynamics through ARHGAP29 and promotes metastasis. Cell Rep. 19, 1495-1502 (2017).

75. Shi, C. et al. Yap promotes hepatocellular carcinoma metastasis and mobilization via governing cofilin/F-actin/lamellipodium axis by regulation of JNK Bnip3/SERCA/CaMKII pathways. Redox Biol. 14, 59-71 (2018).

76. Wang, T. et al. YAP promotes breast cancer metastasis by repressing growth differentiation factor-15. Biochim. Biophys. Acta Mol. Basis Dis. 1864 1744-1753 (2018).
77. Kim, T. et al. MRTF potentiates TEAD-YAP transcriptional activity causing metastasis. EMBO J. 36, 520-535 (2017).

78. Li, C. et al. A ROR1-HER3-IncRNA signalling axis modulates the Hippo-YAP pathway to regulate bone metastasis. Nat. Cell Biol. 19, 106-119 (2017).

79. Kim, J. et al. Long noncoding RNA MALAT1 suppresses breast cancer metastasis. Nat. Genet. 50, 1705-1715 (2018).

80. Kenific, C. M., Thorburn, A. \& Debnath, J. Autophagy and metastasis: another double-edged sword. Curr. Opin. Cell Biol. 22, 241-245 (2010).

81. Avivar-Valderas, A. et al. Regulation of autophagy during ECM detachment is linked to a selective inhibition of mTORC1 by PERK. Oncogene 32, 4932-4940 (2013).

82. Gross, S. R. Actin binding proteins: their ups and downs in metastatic life. Cell Adh. Migr. 7, 199-213 (2013).

83. Chen, W., Bai, Y., Patel, C. \& Geng, F. Autophagy promotes triple negative breast cancer metastasis via YAP nuclear localization. Biochem. Biophys. Res. Commun. 520, 263-268 (2019).

84. Zanconato, F., Cordenonsi, M. \& Piccolo, S. YAP/TAZ at the roots of cancer. Cancer Cell 29, 783-803 (2016).

85. Lu, L. et al. Hippo signaling is a potent in vivo growth and tumor suppressor pathway in the mammalian liver. Proc. Natl Acad. Sci. USA 107, 1437-1442 (2010).

86. Patel, S. H., Camargo, F. D. \& Yimlamai, D. Hippo signaling in the liver regulates organ size, cell fate, and carcinogenesis. Gastroenterology 152 533-545 (2017).

87. Takamura, A. et al. Autophagy-deficient mice develop multiple liver tumors. Genes Dev. 25, 795-800 (2011).

88. Lee, Y. A. et al. Autophagy is a gatekeeper of hepatic differentiation and carcinogenesis by controlling the degradation of Yap. Nat. Commun. 9, 4962 (2018).

89. Wang, P. et al. Activation of Aurora A kinase increases YAP stability via blockage of autophagy. Cell Death Dis. 10, 432 (2019).

90. Zhou, Y. et al. YAP promotes multi-drug resistance and inhibits autophagyrelated cell death in hepatocellular carcinoma via the RAC1-ROS-mTOR pathway. Cancer Cell Int. 19, 179 (2019).

91. Liu, L. \& McKeehan, W. L. Sequence analysis of LRPPRC and its SEC1 domain interaction partners suggests roles in cytoskeletal organization, vesicular trafficking, nucleocytosolic shuttling, and chromosome activity. Genomics 79, 124-136 (2002).

92. Li, W. et al. LRPPRC sustains Yap-P27-mediated cell ploidy and P62-HDAC6mediated autophagy maturation and suppresses genome instability and hepatocellular carcinomas. Oncogene 39, 3879-3892 (2020).

93. Zhang, S. et al. Hippo signaling suppresses cell ploidy and tumorigenesis through Skp2. Cancer Cell 31, https://doi.org/10.1016/j.ccell.2017.04.004 (2017).

94. Klingenberg, M., Matsuda, A., Diederichs, S. \& Patel, T. Non-coding RNA in hepatocellular carcinoma: mechanisms, biomarkers and therapeutic targets. J. Hepatol. 67, 603-618 (2017).

95. Wang, C.-Z., Yan, G.-X., Dong, D.S., Xin, H. \& Liu, Z.-Y. LncRNA-ATB promotes autophagy by activating Yes-associated protein and inducing autophagyrelated protein 5 expression in hepatocellular carcinoma. World J. Gastroenterol. 25, 5310-5322 (2019).

96. Tian, Y. et al. ER-residential Nogo-B accelerates NAFLD-associated HCC mediated by metabolic reprogramming of oxLDL lipophagy. Nat. Commun. 10, 3391 (2019)

97. Chan, D. C. Fusion and fission: interlinked processes critical for mitochondrial health. Annu. Rev. Genet. 46, 265-287 (2012).

98. Hsu, P. et al. Cardiolipin remodeling by TAZ/tafazzin is selectively required for the initiation of mitophagy. Autophagy 11, 643-652 (2015).

99. Chu, C. T. et al. Cardiolipin externalization to the outer mitochondrial membrane acts as an elimination signal for mitophagy in neuronal cells. Nat. Cell Biol. 15, 1197-1205 (2013).

100. Barth, P. G. et al. An X-linked mitochondrial disease affecting cardiac muscle, skeletal muscle and neutrophil leucocytes. J. Neurol. Sci. 62, 327-355 (1983).

101. Ravikumar, B. et al. Regulation of mammalian autophagy in physiology and pathophysiology. Physiol. Rev. 90, 1383-1435 (2010).

102. Chen, H.-T. et al. Crosstalk between autophagy and epithelialmesenchymal transition and its application in cancer therapy. Mol. Cancer 18, 101 (2019).

103. $\mathrm{Xu}$, S. et al. Modulation of autophagy in human diseases strategies to foster strengths and circumvent weaknesses. Med. Res. Rev. 39, 1953-1999 (2019).

104. Barnard, R. A. et al. Autophagy inhibition delays early but not late-stage metastatic disease. J. Pharm. Exp. Ther. 358, 282-293 (2016). 
105. Mulcahy Levy, J. M. \& Thorburn, A. Autophagy in cancer: moving from understanding mechanism to improving therapy responses in patients. Cell Death Differ. 27, 843-857 (2020)

106. Amaravadi, R. K., Kimmelman, A. C. \& Debnath, J. Targeting autophagy in cancer: recent advances and future directions. Cancer Discov. 9, 1167-1181 (2019).

107. Singh, S. S. et al. Dual role of autophagy in hallmarks of cancer. Oncogene 37, 1142-1158 (2018).

108. Giordano, A. \& Tommonaro, G. Curcumin and cancer. Nutrients 11, https:// doi.org/10.3390/nu11102376 (2019).

109. Zhu, J. et al. Curcumin induces autophagy via inhibition of Yes-associated protein (YAP) in human colon cancer cells. Med. Sci. Monit. 24, 7035-7042 (2018).

110. Bai, Z.-L., Tay, V., Guo, S.-Z., Ren, J. \& Shu, M.-G. Silibinin induced human glioblastoma cell apoptosis concomitant with autophagy through simultaneous inhibition of mTOR and YAP. Biomed. Res. Int. 2018, 6165192 (2018).

111. Guo, C. et al. Pharmacological properties and derivatives of shikonin-A review in recent years. Pharm. Res 149, 104463 (2019).

112. Zhu, J., Zhao, L., Luo, B. \& Sheng, W. Shikonin regulates invasion and autophagy of cultured colon cancer cells by inhibiting yes-associated protein Oncol. Lett. 18, 6117-6125 (2019).

113. Booth, L. A., Roberts, J. L. \& Dent, P. The role of cell signaling in the crosstalk between autophagy and apoptosis in the regulation of tumor cell survival in response to sorafenib and neratinib. Semin. Cancer Biol. https://doi.org/ 10.1016/j.semcancer.2019.10.013 (2019)

114. Booth, L., Roberts, J. L., Poklepovic, A. \& Dent, P. The lethality of [pazopanib + HDAC inhibitors] is enhanced by Neratinib. Front. Oncol. 9, 650 (2019).

115. Dent, P. et al. Neratinib inhibits Hippo/YAP signaling, reduces mutant K-RAS expression, and kills pancreatic and blood cancer cells. Oncogene $\mathbf{3 8}$ 5890-5904 (2019).

116. Huang, T. et al. MST4 phosphorylation of ATG4B regulates autophagic activity, tumorigenicity, and radioresistance in glioblastoma. Cancer Cell 32 https://doi.org/10.1016/j.ccell.2017.11.005 (2017).

117. Chen, M. et al. The MST4-MOB4 complex disrupts the MST1-MOB1 complex in the Hippo-YAP pathway and plays a pro-oncogenic role in pancreatic cancer. J. Biol. Chem. 293, 14455-14469 (2018).

118. Dent, P. et al. Neratinib degrades MST4 via autophagy that reduces membrane stiffness and is essential for the inactivation of PI3K, ERK1/2, and YAP/ TAZ signaling. J. Cell Physiol. https://doi.org/10.1002/jcp.29443 (2020).

119. Tang, F. et al. LATS1 but not LATS2 represses autophagy by a kinaseindependent scaffold function. Nat. Commun. 10, 5755 (2019).

120. Kudo, M. et al. Lenvatinib versus sorafenib in first-line treatment of patients with unresectable hepatocellular carcinoma: a randomised phase 3 noninferiority trial. Lancet 391, 1163-1173 (2018).

121. Tai, W. T. et al. Mcl-1-dependent activation of Beclin 1 mediates autophagic cell death induced by sorafenib and SC-59 in hepatocellular carcinoma cells. Cell Death Dis. 4, e485 (2013).

122. Zhang, Y., Whaley-Connell, A. T., Sowers, J. R. \& Ren, J. Autophagy as an emerging target in cardiorenal metabolic disease: From pathophysiology to management. Pharmacol. Ther. 191, https://doi.org/10.1016/j. pharmthera.2018.06.004 (2018)

123. Ge, M. et al. The anti-hepatic fibrosis effects of dihydrotanshinone I are mediated by disrupting the yes-associated protein and transcriptional enhancer factor D2 complex and stimulating autophagy. Br. J. Pharm. 174 1147-1160 (2017).

124. Tang, D. et al. Endogenous HMGB1 regulates autophagy. J. Cell Biol. 190 881-892 (2010).

125. Zhang, Y.-G. et al. Intestinal epithelial HMGB1 inhibits bacterial infection via STAT3 regulation of autophagy. Autophagy 15, 1935-1953 (2019).

126. Zhang, L. et al. Dedifferentiation process driven by radiotherapy-induced HMGB1/TLR2/YAP/HIF-1a signaling enhances pancreatic cancer stemness. Cell Death Dis. 10, 724 (2019).

127. Chen, R. et al. High mobility group protein B1 controls liver cancer initiation through yes-associated protein -dependent aerobic glycolysis. Hepatology 67, 1823-1841 (2018)

128. Hardaway, B. W. Adriamycin-associated cardiomyopathy: where are we now? updates in pathophysiology, dose recommendations, prognosis, and outcomes. Curr. Opin. Cardiol. 34, 289-295 (2019).

129. Luo, P. et al. HMGB1 contributes to adriamycin-induced cardiotoxicity via upregulating autophagy. Toxicol. Lett. 292, 115-122 (2018)
130. Cardinali, D. P. Melatonin: clinical perspectives in neurodegeneration. Front Endocrinol. 10, 480 (2019).

131. Zhang, M. et al. Melatonin protects against diabetic cardiomyopathy through Mst1/Sirt3 signaling. J. Pineal. Res. 63, https://doi.org/10.1111/jpi.12418 (2017).

132. Wang, S. et al. Melatonin activates Parkin translocation and rescues the impaired mitophagy activity of diabetic cardiomyopathy through Mst1 inhibition. J. Cell Mol. Med. 22, 5132-5144 (2018).

133. Komatsu, M. et al. Loss of autophagy in the central nervous system causes neurodegeneration in mice. Nature 441, 880-884 (2006).

134. Shi, L. et al. Melatonin regulates apoptosis and autophagy via ROSMST1 pathway in subarachnoid hemorrhage. Front. Mol. Neurosci. 11, 93 (2018)

135. Yang, Z. \& Klionsky, D. J. Mammalian autophagy: core molecular machinery and signaling regulation. Curr. Opin. Cell Biol. 22, 124-131 (2010).

136. Polson, H. E. J. et al. Mammalian Atg18 (WIPI2) localizes to omegasomeanchored phagophores and positively regulates LC3 lipidation. Autophagy $\mathbf{6}$, 506-522 (2010).

137. Man, S. M. \& Kanneganti, T.-D. Regulation of lysosomal dynamics and autophagy by CTSB/cathepsin B. Autophagy 12, 2504-2505 (2016).

138. Lin, J. et al. Mst1 inhibits CMECs autophagy and participates in the development of diabetic coronary microvascular dysfunction. Sci. Rep. 6, 34199 (2016).

139. You, P. et al. Lin28a protects against diabetic cardiomyopathy through Mst inhibition. J. Cell Physiol. 235, 4455-4465 (2020).

140. Hu, J. et al. Exosomal Mst1 transfer from cardiac microvascular endothelia cells to cardiomyocytes deteriorates diabetic cardiomyopathy. Biochim. Biophys. Acta Mol. Basis Dis. 1864, 3639-3649 (2018).

141. Yuan, P. et al. Laminar flow inhibits the Hippo/YAP pathway via autophagy and SIRT1-mediated deacetylation against atherosclerosis. Cell Death Dis. 11, 141 (2020).

142. Wang, T. et al. Mst1 participates in the atherosclerosis progression through macrophage autophagy inhibition and macrophage apoptosis enhancement. J. Mol. Cell Cardiol. 98, 108-116 (2016).

143. Shang, $X$. et al. Mst1 deletion reduces septic cardiomyopathy via activating Parkin-related mitophagy. J. Cell Physiol. 235, 317-327 (2020).

144. Yu, W., Xu, M., Zhang, T., Zhang, Q. \& Zou, C. Mst1 promotes cardiac ischemiareperfusion injury by inhibiting the ERK-CREB pathway and repressing FUNDC1-mediated mitophagy. J. Physiol. Sci. 69, 113-127 (2019).

145. Yao, L. et al. Regulation of YAP by mammalian target of rapamycin complex 1 in endothelial cells controls blood pressure through COX-2/mPGES-1/PGE cascade. Hypertension 74, 936-946 (2019).

146. Lee, J. K et al. MST1 functions as a key modulator of neurodegeneration in a mouse model of ALS. Proc. Natl Acad. Sci. USA 110, 12066-12071 (2013).

147. Zhang, M., Tao, W., Yuan, Z. \& Liu, Y. Mst-1 deficiency promotes posttraumatic spinal motor neuron survival via enhancement of autophagy flux. J. Neurochem. 143, 244-256 (2017).

148. Xiao, D. et al. Enhanced mitophagy mediated by the YAP/Parkin pathway protects against DOX-induced cardiotoxicity. Toxicol. Lett. 330, https://doi. org/10.1016/j.toxlet.2020.05.015 (2020).

149. Zhou, T., Chang, L., Luo, Y., Zhou, Y. \& Zhang, J. Mst1 inhibition attenuates non-alcoholic fatty liver disease via reversing Parkin-related mitophagy. Redox Biol. 21, 101120 (2019)

150. Liu, Z. et al. A potential role for the Hippo pathway protein, YAP, in controlling proliferation, cell cycle progression, and autophagy in BCPAP and KI thyroid papillary carcinoma cells. Am. J. Transl. Res. 9, 3212-3223 (2017).

151. Li, L. et al. Deacetylation of tumor-suppressor MST1 in Hippo pathway induces its degradation through HBXIP-elevated HDAC6 in promotion of breast cancer growth. Oncogene 35, 4048-4057 (2016).

152. Zhang, J. et al. Overexpression of macrophage stimulating 1 enhances the anti-tumor effects of IL-24 in esophageal cancer via inhibiting ERK-Mfn2 signaling-dependent mitophagy. Biomed. Pharmacother. 114, 108844 (2019).

153. Fan, S. et al. PINK1-dependent mitophagy regulates the migration and homing of multiple myeloma cells via the MOB1B-mediated Hippo-YAP/TAZ pathway. Adv. Sci. 7, 1900860 (2020).

154. Hu, Y. et al. Mammalian STE20-like kinase 1 regulates pancreatic cancer cell survival and migration through Mfn2-mediated mitophagy. Mol. Med. Rep. 22, 398-404 (2020).

155. Wei, R. et al. FAT4 regulates the EMT and autophagy in colorectal cancer cells in part via the PI3K-AKT signaling axis. J. Exp. Clin. Cancer Res. 38, 112 (2019). 